\title{
When do Foxby classes coincide with the classes of modules of finite Gorenstein dimensions?
}

\author{
Driss Bennis, J. R. García Rozas, and Luis Oyonarte
}

\begin{abstract}
The relation between the Auslander (resp., Bass) class and the class of modules with finite Gorenstein projective (resp., injective) dimension is well known when these mentioned classes are built with a dualizing module over Noetherian $n$-perfect rings. Basically, the results are necessary conditions to ensure that both classes coincide. In this article we try to extend and sometimes improve some of these results by weakening the condition of being dualizing. Among other results, we prove that a Wakamatsu tilting module with some extra conditions is precisely a module ${ }_{R} C$ such that the Bass class $\mathcal{B}_{C}(R)$ coincides with the class of modules of finite Gorenstein injective dimension.
\end{abstract}

\section{Introduction}

It is a classical result that any module over a Gorenstein ring has finite Gorenstein injective and projective dimensions (see [4]). However, to study modules of finite Gorenstein dimension over more general rings, one has to restrict to some full subcategories. For this purpose, Auslander and Bass classes have been proved to be suitable full subcategories. Namely, Foxby [8] introduced a duality between these two full subcategories over a commutative local Cohen-Macaulay ring admitting a dualizing module. He proved that the finitely generated modules in the Auslander class are precisely those of finite Gorenstein projective dimension. Enochs, Jenda, and Xu [6] extended this result to modules which are not necessarily finitely generated and also proved the dual result. The main result of that paper is that the two classes involved in Foxby's duality coincide with the classes of those modules having finite Gorenstein projective dimension and those having finite Gorenstein injective dimension.

Enochs, Jenda, and López-Ramos proved in [5] that, over a (not necessarily commutative) Noetherian $n$-perfect ring with a dualizing bimodule, the Auslander class (resp., Bass class) coincides with the class of modules with finite

Kyoto Journal of Mathematics, Vol. 56, No. 4 (2016), 785-802 DOI $10.1215 / 21562261-3664914$, (C) 2016 by Kyoto University

Received March 23, 2015. Accepted October 6, 2015.

2010 Mathematics Subject Classification: Primary 16E30; Secondary 18G25.

Garcia Rozas's and Oyonarte's work was partially supported by the grant MTM2014-54439 from Ministerio de Ciencia e Innovación of Spain. 
Gorenstein projective (resp., injective) dimension. Holm and White [11] continued the study of the noncommutative case considering a semidualizing bimodule. (Enochs and Yassemi [7] also considered semidualizing ones in the commutative setting.)

Our goal in this article is twofold. First we aim to generalize the previous results, and also we aim to study the converse problem. Namely, if $C$ is any left $R$-module and $S=\operatorname{End}_{R}(C)$, then one can consider the Auslander $\mathcal{A}_{C}(S)$ and the Bass $\mathcal{B}_{C}(R)$ classes associated to $C$. Thus, our first concern is the following question: under what conditions on $C$ do these classes coincide with the classes of modules with finite Gorenstein projective and injective dimensions, respectively? We will see that $C$ is close to being a semidualizing bimodule (see Theorems 3.4 and 3.7). On the other hand, the $C$-Gorenstein projective and $C$-Gorenstein injective modules, introduced by Geng and Ding [10], are of interest. We will show, in Theorems 3.4 and 3.7, that the Auslander class (resp., Bass class) coincides with the class of modules with finite $C$-Gorenstein injective (resp., projective) dimension, under some conditions on $C$ (close to the ones of a dualizing bimodule over its endomorphism ring). The second aim of this article is to investigate when the Auslander and Bass classes are covering and enveloping.

The article is organized as follows. In Section 2 we give some terminology and some preliminary results. Namely, we recall the concept of a Wakamatsu tilting module and the definitions of Auslander and Bass classes. We also recall some results from [1, Section 5] that will be used in the next sections. Of some importance will be the concepts of Hom-faithful and Hom-cofaithful modules relative to a class of modules.

In Section 3 we characterize the self-Hom-faithful left $R$-modules $C$ with right Noetherian and left $n$-perfect endomorphism $\operatorname{ring} S$ verifying $\operatorname{inj} . \operatorname{dim}_{R}(C)<\infty$ and inj. $\operatorname{dim}_{S}(C)<\infty$ such that the Auslander class $\mathcal{A}_{C}(S)$ coincides with the class of left $S$-modules with finite Gorenstein projective dimension. These modules are precisely those ${ }_{R} C$ that are $\Sigma$-self-orthogonal and self-small (Theorem 3.4). It turns out that this is equivalent to the condition that the Bass class $\mathcal{B}_{C}(R)$ coincides with the class of modules with a finite left $C$ - $G P(R)$-resolution, where $C-G P(R)$ is the class of $C$-Gorenstein projective modules. (These were introduced by Geng and Ding [10].) A dual version of that result is Theorem 3.7. As a corollary of the last theorem, we prove that a Wakamatsu tilting module with some extra conditions is precisely a left $R$-module $C$ such that the Bass class $\mathcal{B}_{C}(R)$ coincides with the class of modules of finite Gorenstein injective dimension (Corollary 3.9). We end Section 3 with the classical Foxby duality with respect to left $R$-modules $C$ that are more general than (semi)dualizing ones (Corollary 3.10).

In Section 4, we apply the results in Sections 2 and 3 to the problem of the existence of (pre)covers and (pre)envelopes relative to the class of modules with finite Gorenstein projective and injective dimensions (Theorems 4.5 and 4.6). 


\section{Preliminaries}

Throughout this article, $R$ will be an associative (not necessarily commutative) ring with identity, and all modules will be, unless otherwise specified, unital left $R$-modules. When right $R$-modules need to be used, they will be denoted by $M_{R}$, while left $R$-modules will be denoted by ${ }_{R} M$. Under this terminology, ${ }_{R} M_{S}$ will mean that $M$ is an $(R, S)$-bimodule. We use $\operatorname{Proj}(R)(\operatorname{resp} ., \operatorname{Inj}(R)$ and $\operatorname{Flat}(R))$ to denote the class of all projective (resp., injective and flat) $R$-modules. The category of all left (resp., right if needed) $R$-modules will be denoted by $R$ Mod (resp., Mod- $R$ ). For an $R$-module $C$ we use $\operatorname{Add}_{R}(C)\left(\operatorname{resp}\right.$., $\left.\operatorname{add}_{R}(C)\right)$ to denote the class of all $R$-modules which are isomorphic to direct summands of direct sums (resp., finite direct sums) of copies of $C$, and $\operatorname{Prod}_{R}(C)$ will denote the class of all $R$-modules which are isomorphic to direct summands of direct products of copies of $C$. By $\sigma_{R}[C]$ we mean the full subcategory of $R$-Mod of modules subgenerated by $C$, that is, isomorphic to submodules of quotients of direct sums of arbitrary copies of $C$. Dually, let $\pi_{R}[C]$ be the class of left $R$ modules which are quotients of submodules of direct products of copies of $C$ (see [15]).

Given a class of modules $\mathcal{F}$ (which will always be considered closed under isomorphisms), an $\mathcal{F}$-precover of $M \in R$-Mod is a morphism $\varphi: F \rightarrow M(F \in \mathcal{F})$ such that $\operatorname{Hom}_{R}\left(F^{\prime}, \varphi\right)$ is surjective for every $F^{\prime} \in \mathcal{F}$. If, in addition, any solution of the equation $\operatorname{Hom}_{R}(F, \varphi)(g)=\varphi$ is an automorphism of $F$, then $\varphi$ is said to be an $\mathcal{F}$-cover. The $\mathcal{F}$-precover $\varphi$ is said to be special if it is surjective and $\operatorname{Ext}^{1}(F, \operatorname{ker} \varphi)=0$ for every $F \in \mathcal{F}$. We can define $\mathcal{F}$-(special pre)envelopes dually. The class $\mathcal{F}$ is said to be (pre)covering (resp., (pre)enveloping) if every module has an $\mathcal{F}$-(pre)cover (resp., $\mathcal{F}$-(pre)envelope).

Given the class $\mathcal{F}$, the class of all modules $N$ such that $\operatorname{Ext}_{R}{ }_{R}^{1}(F, N)=0$ for every $F \in \mathcal{F}$ will be denoted by $\mathcal{F}^{\perp}$. (Similarly, ${ }^{\perp} \mathcal{F}=\left\{N \mid \operatorname{Ext}_{\bar{R}}^{\geq 1}(N, F)=0\right.$ $\forall F \in \mathcal{F}\}$.)

A left $\mathcal{F}$-resolution of an $R$-module $M$ is a complex (so not necessarily exact) of $R$-modules in $\mathcal{F}$,

$$
\mathbf{X}=\cdots \rightarrow X_{1} \rightarrow X_{0} \rightarrow M \rightarrow 0,
$$

such that $\operatorname{Hom}_{R}(F, \mathbf{X})$ is an exact complex for every $F \in \mathcal{F}$. Thus, we see that $M$ having a left $\mathcal{F}$-resolution is equivalent to $M$ having an $\mathcal{F}$-precover whose kernel has an $\mathcal{F}$-precover and so on. Right $\mathcal{F}$-resolutions may be defined dually.

Recall (see [14]) that ${ }_{R} C$ is Wakamatsu tilting if the following hold.

1. There is an exact sequence

$$
\cdots \rightarrow P^{1} \rightarrow P^{0} \rightarrow C \rightarrow 0
$$

with all the $P^{i}$ 's finitely generated projective left $R$-modules (i.e., ${ }_{R} C$ has a degreewise finite projective resolution).

2. $\operatorname{Ext}_{\bar{R}}^{\geq 1}(C, C)=0$. 
3. There exists an exact sequence of $R$-modules

$$
\mathbf{X}: 0 \longrightarrow R \stackrel{f_{0}}{\longrightarrow} C_{0} \stackrel{f_{1}}{\longrightarrow} C_{1} \stackrel{f_{2}}{\longrightarrow} C_{2} \stackrel{f_{3}}{\longrightarrow} \cdots,
$$

where, for every $i \in \mathbb{N}, C_{i} \in \operatorname{add}(C)$, such that $\operatorname{Hom}_{R}(-, C)$ leaves the sequence $\mathbf{X}$ exact.

In this article $C$ will always be a left $R$-module with endomorphism ring $S=\operatorname{End}_{R}(C)$. Throughout this section we will present some results that will be needed in the subsequent sections. They have been already proved in $[1$, Section 5], nevertheless, so we include them here (without proof) for the reader's convenience.

Associated to the bimodule ${ }_{R} C_{S}$ we have the Auslander and Bass classes (also called Foxby classes by other authors), $\mathcal{A}_{C}(S)$ and $\mathcal{B}_{C}(R)$, respectively, defined as follows (see, e.g., [11]).

The Auslander class $\mathcal{A}_{C}(S)$ consists of all left $S$-modules $M$ satisfying:

(A1) $\operatorname{Tor}_{\geq 1}^{S}(C, M)=0$,

(A2) $\operatorname{Ext}_{\bar{R}} \geq 1\left(C, C \otimes_{S} M\right)=0$,

(A3) the canonical map $\mu_{M}: M \rightarrow \operatorname{Hom}_{R}\left(C, C \otimes_{S} M\right)$ is an isomorphism of $S$-modules.

The Bass class $\mathcal{B}_{C}(R)$ consists of all left $R$-modules $N$ satisfying:

(B1) $\operatorname{Ext}_{R}^{\geq 1}(C, N)=0$,

(B2) $\operatorname{Tor}_{\geq 1}^{S}\left(C, \operatorname{Hom}_{R}(C, N)\right)=0$,

(B3) the canonical map $\nu_{N}: C \otimes_{S} \operatorname{Hom}_{R}(C, N) \rightarrow N$ is an isomorphism of $R$-modules.

\section{DEFINITION 2.1}

A left $R$-module $M$ is said to be Hom-faithful relative to a class of left $R$-modules $\mathcal{C}$ if $\operatorname{Hom}_{R}(M, N)=0, N \in \mathcal{C} \Rightarrow N=0$. We will say simply that $M$ is Hom-faithful if $M$ is Hom-faithful relative to $R$-Mod.

A left $R$-module $M$ is said to be self-Hom-faithful if $M$ is Hom-faithful relative to $\sigma[M]$.

A left $R$-module $M$ is said to be Hom-cofaithful relative to a class of left $R$-modules $\mathcal{C}$ if $\operatorname{Hom}_{R}(N, M)=0, N \in \mathcal{C} \Rightarrow N=0$. We will say simply that $M$ is Hom-cofaithful if $M$ is Hom-cofaithful relative to $R$-Mod.

A left $R$-module $M$ is said to be self-Hom-cofaithful if $M$ is Hom-faithful relative to $\pi[M]$.

A right $S$-module $N$ is said to be $\otimes_{S}$-faithful relative to a class $\mathcal{D}$ of left $S$-modules if $N \otimes_{S} M=0, M \in \mathcal{D} \Rightarrow M=0$. We will say simply that $N$ is $\otimes_{S^{-}}$ faithful if $N$ is $\otimes_{S}$-faithful relative to $S$-Mod.

A left $R$-module $M$ is self-small if $\operatorname{Hom}_{R}\left(M, M^{(I)}\right) \cong \operatorname{Hom}_{R}(M, M)^{(I)}$ for every set $I$. 
We use the term faithful to describe these kinds of modules since, over a commutative $\operatorname{ring} R$, a finitely presented $R$-module $C$ with $\operatorname{Ann}_{R}(C)=0$ (i.e., a faithful module in the terminology of commutative algebra) is Hom-faithful in our sense (see [11, Proposition 3.6]).

Let $E$ be an injective cogenerator in $R$-Mod, and let $C^{\vee}=\operatorname{Hom}_{R}(C, E)$.

\section{LEMMA 2.2}

The following statements hold.

(a) If $C_{S}$ is Hom-faithful relative to $\left.\mathcal{D}^{+}=\operatorname{Hom}_{\mathbb{Z}}(X, \mathbb{Q} / \mathbb{Z}): X \in \mathcal{D}\right\}$, then it is $\otimes_{S}$-faithful relative to $\mathcal{D}$.

(b) If $C_{S}$ is finitely presented and $\otimes_{S}$-faithful relative to $\mathcal{D}$, then $C_{S}$ is Homfaithful relative to $\mathcal{D}^{+}$.

(c) $C$ is $\otimes_{S}$-faithful relative to $\pi_{S}\left[C^{\vee}\right]$ if and only if $C^{\vee}$ is self-Homcofaithful in $S$-Mod.

\section{Proof}

For (a) and (b) see [1, Lemma 5.2]. It is easy to show (c).

In what follows we will often need the module $C$ to meet some conditions relative to the cancellation of the Ext functor. Namely, we will need $\operatorname{Ext}_{\bar{R}}^{\geq 1}\left(C, C^{(I)}\right)=0$ for every index set $I$ (i.e., $C^{(I)} \in \operatorname{Add}_{R}(C)^{\perp}$ for every index set $I$ ). This type of module has already been used by several authors, and they exist in the literature as $\Sigma$-self-orthogonal modules.

\section{PROPOSITION 2.3}

The following statements hold.

1. Every projective left $S$-module lies in $\mathcal{A}_{C}(S)$ if and only if $C$ is a $\Sigma$-selforthogonal and self-small left $R$-module.

2. If ${ }_{R} C$ is self-Hom-faithful, then $\mathcal{A}_{C}(S)$ is closed under cokernels of monomorphisms.

3. If ${ }_{R} C$ is a self-Hom-faithful, $\Sigma$-self-orthogonal, and self-small left $R$ module, then for every left $S$-module $M$ we have: $\operatorname{proj}_{\operatorname{dim}}(M)<\infty \Rightarrow M \in$ $\mathcal{A}_{C}(S)$.

4. $\mathcal{A}_{C}(S)$ is closed under kernels of epimorphisms and under extensions.

Proof

See [1, Proposition 5.4].

We will denote by $\overline{\operatorname{Add}_{R}(C)}$ the class of left $R$-modules with a finite exact right $\operatorname{Add}_{R}(C)$-resolution.

Dually we have the following. 
PROPOSITION 2.4

The following statements hold.

1. If ${ }_{R} C$ is $\Sigma$-self-orthogonal and self-small, then $\operatorname{Add}_{R}(C) \subseteq \mathcal{B}_{C}(R)$. If, in addition, $C^{\vee}$ is self-Hom-cofaithful, then the converse holds too.

2. If $C^{\vee}$ is self-Hom-cofaithful, then $\mathcal{B}_{C}(R)$ is closed under kernels of epimorphisms.

3. If $C^{\vee}$ is a self-Hom-cofaithful, $\Sigma$-self-orthogonal, and self-small left $R$ module, then for every left $R$-module $M$ we have $M \in \overline{\operatorname{Add}_{R}(C)} \Rightarrow M \in \mathcal{B}_{C}(R)$.

4. $\mathcal{B}_{C}(R)$ is closed under cokernels of monomorphisms and under extensions.

Proof

See [1, Proposition 5.6].

THEOREM 2.5

If $C_{S}$ has a degreewise finite projective resolution, then the following assertions are equivalent:

(i) $\operatorname{Inj}(R) \subseteq \mathcal{B}_{C}(R)$;

(ii) (a) ${ }_{R} C$ is balanced, that is, the canonical map $R \rightarrow \operatorname{End}_{S}(C)$ is an isomorphism, and

(b) $C_{S}$ is self-orthogonal, that is, $\operatorname{Ext}_{\bar{S}}^{\geq 1}(C, C)=0$.

If, in addition, ${ }_{R} C$ is Hom-faithful relative to $\sigma[C] \cup\{E / K: K \leq E\}$, (i) and (ii) are equivalent to

(iii) $\operatorname{Prod}\left(C^{\vee}\right) \subseteq \mathcal{A}_{C}(S)$.

Proof

See [1, Theorem 5.9].

\section{Foxby classes and Gorenstein dimensions}

Throughout this section we will use the symbols $\mathcal{G I}(R)$ (resp., $\mathcal{G P}(R)$ ) to denote the class of all Gorenstein injective (resp., projective) $R$-modules. The Gorenstein injective (resp., projective) dimension of a module $M$ will be denoted as $\operatorname{Gid}(M)$ (resp., $\operatorname{Gpd}(M))$.

From now on we fix an injective cogenerator $E$ in $R$-Mod and consider the left $S$-module $C^{\vee}=\operatorname{Hom}_{R}(C, E)$.

\section{DEFINITION 3.1}

The class of all syzygies of exact complexes of left $R$-modules in $\operatorname{Add}_{R}(C)$ which, in addition, are $\operatorname{Hom}_{R}\left(\operatorname{Add}_{R}(C),-\right)$-exact and $\operatorname{Hom}_{R}\left(-, \operatorname{Add}_{R}(C)\right)$-exact, will be denoted as $C-G P(R)$. 
Dually, $C$ - $G I(R)$ will be the class of all syzygies of exact, $\operatorname{Hom}_{R}\left(\operatorname{Prod}_{R}\left(C^{\vee}\right)\right.$, -)-exact, and $\operatorname{Hom}_{R}\left(-, \operatorname{Prod}_{R}\left(C^{\vee}\right)\right)$-exact complexes of left $R$-modules with components in $\operatorname{Prod}_{R}\left(C^{\vee}\right)$.

\section{PROPOSITION 3.2}

Let $C$ be a left $R$-module.

(a) If $\operatorname{Inj}(R) \subseteq \mathcal{B}_{C}(R)$ and inj. $\operatorname{dim}_{R}(C)<\infty$, then $\mathcal{G I}(R) \subseteq \mathcal{B}_{C}(R)$.

(b) If $\operatorname{Proj}(S) \subseteq \mathcal{A}_{C}(S)$ and there is an injective cogenerator $E$ in $R$-Mod such that proj. $\operatorname{dim}_{S}\left(\operatorname{Hom}_{R}(C, E)\right)<\infty$, then $\mathcal{G P}(S) \subseteq \mathcal{A}_{C}(S)$.

Proof

(a) Let $M \in \mathcal{G I}(R)$. Then $M=\operatorname{ker}\left(I_{0} \rightarrow I_{1}\right)$ where

$$
\cdots \rightarrow I^{1} \rightarrow I^{0} \rightarrow I_{0} \rightarrow I_{1} \rightarrow \cdots
$$

is a complete injective resolution. Since inj. $\operatorname{dim}_{R}(C)<\infty$, the sequence above is $\operatorname{Hom}_{R}(C,-)$-exact, and hence, $\operatorname{Ext}_{R}^{i \geq 1}(C, M)=0$ and we get (B1).

From the above we have an exact left $\operatorname{Add}_{R}(C)$-resolution

$$
\cdots \rightarrow I^{1} \rightarrow I^{0} \rightarrow M \rightarrow 0
$$

from which we get a commutative diagram

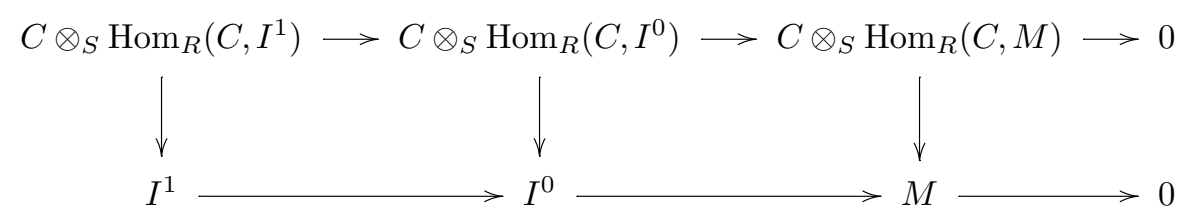

where the first two vertical arrows are isomorphisms since $\operatorname{Inj}(R) \subseteq \mathcal{B}_{C}(R)$. Thus, the third vertical arrow is an isomorphism too and we have (B3). Finally, (B2) is also obtained from the diagram since

$$
\operatorname{Tor}_{i \geq 1}^{S}\left(C, \operatorname{Hom}_{R}\left(C, I^{j}\right)\right)=0, \quad j \geq 1,
$$

by the condition $\operatorname{Inj}(R) \subseteq \mathcal{B}_{C}(R)$. Therefore, $M \in \mathcal{B}_{C}(R)$.

(b) Let $N \in \mathcal{G P}(S)$. Then $N=\operatorname{ker}\left(P_{0} \rightarrow P_{1}\right)$, where

$$
\cdots \rightarrow P^{1} \rightarrow P^{0} \rightarrow P_{0} \rightarrow P_{1} \rightarrow \cdots
$$

is a complete projective resolution.

By the hypothesis, the sequence is $\operatorname{Hom}_{S}\left(-, \operatorname{Hom}_{R}(C, E)\right)$-exact, that is, $\operatorname{Hom}_{R}\left(C \otimes_{S}-, E\right)$-exact, that is, $\left(C \otimes_{S}-\right)$-exact. This means that $\operatorname{Tor}_{i \geq 1}^{S}(C$, $N)=0$ and so we get (A1). 
Finally, from the exact sequence $0 \rightarrow N \rightarrow P^{0} \rightarrow P^{1}$ we have the commutative diagram

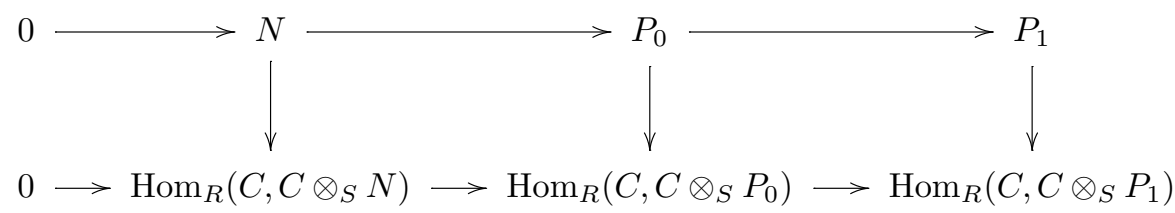

where the last two vertical arrows are isomorphisms since $\operatorname{Proj}(S) \subseteq \mathcal{A}_{C}(S)$. Thus, the first vertical arrow is an isomorphism too and we have (A3).

Again, (A2) is obtained from the diagram since $\operatorname{Ext}_{R}^{i \geq 1}\left(C, C \otimes_{S} P_{i}\right)=0, i \geq 0$, because $\operatorname{Proj}(S) \subseteq \mathcal{A}_{C}(S)$. Therefore, $N \in \mathcal{A}_{C}(S)$.

Recall from [5] that a ring $R$ is said to be left $n$-perfect if every flat left $R$-module has projective dimension at most $n$.

\section{LEMMA 3.3}

Let $S$ be a right coherent and left n-perfect ring. Then the class $\mathcal{L}(S)$ of left $S$-modules of finite projective dimension is preenveloping.

\section{Proof}

By the hypothesis, $\mathcal{L}(S)$ is closed under products. The result now follows by standard arguments of preenvelopes (see [13]).

The next result generalizes [6, Theorem 2.1], [6, Proposition 1.3], and [5, Corollary 3.19].

\section{THEOREM 3.4}

Let ${ }_{R} C$ be a left $R$-module, and let $S=\operatorname{End}_{R}(C)$. Suppose that

(a.1) inj. $\operatorname{dim}_{R}(C)=d<\infty$.

(a.2) inj. $\operatorname{dim}_{S}(C)<\infty$.

(b) ${ }_{R} C$ is self-Hom-faithful.

(c) $S$ is right Noetherian and left $n$-perfect.

Then, the following conditions are equivalent.

(i) ${ }_{R} C$ is $\Sigma$-self-orthogonal and self-small.

(ii) $\mathcal{A}_{C}(S)=\{M \in S$-Mod $\mid \operatorname{Gpd}(M)<\infty\}$.

(iii) $\mathcal{B}_{C}(R)$ coincides with the class of modules with a finite left $C$-GP $(R)$ resolution.

\section{Proof}

(ii) $\Rightarrow$ (i) This follows by Proposition 2.3(1).

(i) $\Rightarrow$ (ii) By Proposition 2.3(1) we have $\operatorname{Proj}(S) \subseteq \mathcal{A}_{C}(S)$. By Proposition $3.2(\mathrm{~b}), \mathcal{G P}(S) \subseteq \mathcal{A}_{C}(S)$. (Note that, by (a.2) and (c), $\operatorname{Hom}_{R}(C, I)$ has finite 
projective dimension as a left $S$-module for all $I \in \operatorname{Inj}(R)$ by [12, Theorem 1.5].) Therefore, by Proposition 2.3(2), $\{M \in S$-Mod $\mid \operatorname{Gpd}(M)<\infty\} \subseteq \mathcal{A}_{C}(S)$.

Now let $N \in \mathcal{A}_{C}(S)$, and let

$$
\cdots \rightarrow P^{1} \rightarrow P^{0} \rightarrow N \rightarrow 0
$$

be a projective resolution. We will see that $K^{d-1}=\operatorname{ker}\left(P^{d-1} \rightarrow P^{d-2}\right)$ is Gorenstein projective and so $\operatorname{Gpd}(N)<\infty$.

The sequence

$$
\cdots \rightarrow C \otimes_{S} P^{0} \rightarrow C \otimes_{S} N \rightarrow 0
$$

is exact by (A1). Also we see by the hypothesis that $\operatorname{Ext}_{R}^{i \geq 1}\left(C \otimes_{S} P, C \otimes_{S} Q\right)=0$ for all $P, Q \in \operatorname{Proj}(S)$. Now, by (a.1), inj. $\operatorname{dim}_{R}\left(C \otimes_{S} P\right) \leq d, \forall P \in \operatorname{Proj}(S)$. Hence, the last sequence is $\operatorname{Hom}_{R}\left(-, C \otimes_{S} \operatorname{Proj}(S)\right)$-exact from the term $C \otimes_{S} P^{d}$ to the left. But

$$
\operatorname{Hom}_{R}\left(C \otimes_{S} P^{i}, C \otimes_{S} P\right) \cong \operatorname{Hom}_{S}\left(P^{i}, \operatorname{Hom}_{R}\left(C, C \otimes_{S} P\right)\right) \cong \operatorname{Hom}_{S}\left(P^{i}, P\right),
$$

and so the sequence

$$
\operatorname{Hom}_{S}\left(P^{d}, P\right) \rightarrow \operatorname{Hom}_{S}\left(P^{d+1}, P\right) \rightarrow \cdots
$$

is exact. Therefore, $\operatorname{Ext}_{S}^{i \geq d+1}(N, P)=0$, and hence, $\operatorname{Ext}_{S}^{i \geq 1}\left(K^{d-1}, P\right)=0$, so

$$
\operatorname{Ext}_{S}^{i \geq 1}\left(K^{d-1}, L\right)=0
$$

if proj. $\operatorname{dim}_{S}(L)<\infty$.

By (c) we see that the conditions of Lemma 3.3 are satisfied so all left $S$ modules have a $\mathcal{L}(S)$-preenvelope. Then let $K^{d-1} \rightarrow L$ be an $\mathcal{L}(S)$-preenvelope (which is a monomorphism since $K^{d-1} \subseteq P^{d-1}$ ), and consider a sequence

$$
0 \rightarrow T \rightarrow Q^{0} \rightarrow L \rightarrow 0
$$

with $Q^{0}$ projective and $T \in \mathcal{L}(S)$. Thus, $K^{d-1} \rightarrow L$ factors through a morphism $K^{d-1} \rightarrow Q^{0}$ which is still an $\mathcal{L}(S)$-preenvelope, so we have an exact sequence

$$
0 \rightarrow K^{d-1} \rightarrow Q^{0} \rightarrow B^{0} \rightarrow 0
$$

with $\operatorname{Ext}_{S}^{i \geq 1}\left(B^{0}, P\right)=0$ for all $P \in \operatorname{Proj}(S)$. By Proposition 2.3(4), $K^{d-1} \in$ $\mathcal{A}_{C}(S)$ and then, by Proposition 2.3(2) (using hypothesis (b)), $B^{0} \in \mathcal{A}_{C}(S)$.

Let $C \otimes_{S} B^{0} \rightarrow I$ be an injective envelope in $R$-Mod. Then $B^{0} \cong \operatorname{Hom}_{R}(C$, $\left.C \otimes_{S} B^{0}\right) \subseteq \operatorname{Hom}_{R}(C, I)$, which lies in $\mathcal{L}(S)$ by (a.2) and (c) (applying [12, Theorem 1.5]). We can continue in the same manner with $B^{0}$ and get $Q^{1}$, and so on. So we have an exact sequence

$$
0 \rightarrow K^{d-1} \rightarrow Q^{0} \rightarrow Q^{1} \rightarrow \cdots
$$

with $Q^{i} \in \operatorname{Proj}(S)$, which is $\operatorname{Hom}_{S}(-, \operatorname{Proj}(S))$-exact. Then, since

$$
\operatorname{Ext}_{S}^{i \geq 1}\left(K^{d-1}, P\right)=0, \quad \forall P \in \operatorname{Proj}(S),
$$

we conclude that $K^{d-1} \in \mathcal{G P}(S)$.

(ii) $\Rightarrow$ (iii) By the equivalence of categories $C \otimes_{S}-: \mathcal{A}_{C}(S) \rightarrow \mathcal{B}_{C}(R)$ we have $\mathcal{B}_{C}(R)=\left\{C \otimes_{S} M \mid \operatorname{Gpd}(M)<\infty\right\}$. It can be seen that $C \otimes_{S} M$ with 
$\operatorname{Gpd}(M)<\infty$ has a finite left $C$ - $G P(R)$-resolution by simply showing that $C \otimes_{S}$ $G$ is in $C-G P(R)$ for any Gorenstein projective left $S$-module $G$ and that $C \otimes_{S}-$ makes exact any Gorenstein projective left resolution of a module (necessarily) in $\mathcal{A}_{C}(S)$. We see the first one. Let $P^{\bullet}$ be a complete projective resolution of $G$; then $C \otimes_{S} P^{\bullet}$ is again an exact complex since $\mathcal{G P}(S) \subseteq \mathcal{A}_{C}(S)$. The components of this complex are in $\operatorname{Add}(C)$. Now $\operatorname{Hom}_{R}\left(C, C \otimes_{S} P^{\bullet}\right) \cong P^{\bullet}$ since ${ }_{R} C$ is self-small and $\operatorname{Hom}_{R}(C, C)=S$. Hence, $C \otimes_{S} P^{\bullet}$ is $\operatorname{Hom}_{R}(\operatorname{Add}(C),-)$-exact. Also $\operatorname{Hom}_{R}\left(C \otimes_{S}\right.$ $\left.P^{\bullet}, C^{(X)}\right) \cong \operatorname{Hom}_{S}\left(P^{\bullet}, \operatorname{Hom}_{R}\left(C, C^{(X)}\right)\right) \cong \operatorname{Hom}_{S}\left(P^{\bullet}, S^{(X)}\right)$, where the last isomorphism is given since ${ }_{R} C$ is self-small. The last complex is exact due to $P^{\bullet}$ being a complete projective resolution; therefore, $C \otimes_{S} P^{\bullet}$ is $\operatorname{Hom}_{R}(-, \operatorname{Add}(C))$ exact. Finally, let

$$
0 \rightarrow G^{n} \rightarrow G^{n-1} \rightarrow \cdots \rightarrow G^{0} \rightarrow M \rightarrow 0
$$

be a Gorenstein projective left resolution of ${ }_{S} M$. The sequence

$$
0 \rightarrow C \otimes_{S} G^{n} \rightarrow C \otimes_{S} G^{n-1} \rightarrow \cdots \rightarrow C \otimes_{S} G^{0} \rightarrow C \otimes_{S} M \rightarrow 0
$$

is exact since $\operatorname{Tor}_{i}^{S}(C, K)=0$ for any syzygy $K$ in the first sequence. (Note that any such syzygy is in $\mathcal{A}_{C}(S)$.) Therefore, $C \otimes_{S} M$ has a finite left $C$-GP(R)resolution.

On the other hand, since $C-G P(R) \subseteq \mathcal{B}_{C}(R)$ and $\mathcal{B}_{C}(R)$ is closed under cokernels of monomorphisms, we get that the class of modules with a finite left $C-G P(R)$-resolution is contained in $\mathcal{B}_{C}(R)$.

(iii) $\Rightarrow$ (ii) This is the reverse argument of the above.

\section{EXAMPLE 3.5}

It is easy to find an example of a nondualizing module that verifies the conditions of Theorem 3.4. Let $R$ be the $2 \times 2$ upper-triangular matrix ring over a field $K$. Let $C$ be the simple left $R$-module which is obtained by making the quotient of the second column by the Jacobson radical. Then ${ }_{R} C$ is injective, and since End $_{R}(C) \cong K$, we have trivially that $C_{S}$ is injective and that $S$ is left Noetherian and right $n$-perfect. Note that $C$ is self-Hom-faithful since $\sigma_{R}[C]$ is the class of modules with socle given by direct sums of copies of $C$. Also note that $\operatorname{End}_{S}(C) \cong$ $K$, which is not isomorphic to $R$.

\section{EXAMPLE 3.6}

We can get a family of examples of non-(semi)dualizing modules verifying the conditions of Theorem 3.4 by considering the triangular matrix ring

$$
R=\left(\begin{array}{cc}
\mathbb{Z}[G] & \mathbb{Q}[G] \\
0 & \mathbb{Q}[G]
\end{array}\right),
$$

where $G$ is a finite group, and the idempotent

$$
e=\left(\begin{array}{ll}
1 & 0 \\
0 & 0
\end{array}\right) .
$$


Let

$$
C=R e=\left(\begin{array}{cc}
\mathbb{Z}[G] & 0 \\
0 & 0
\end{array}\right) .
$$

The endomorphism ring is

$$
S=e R e \cong \mathbb{Z}[G] .
$$

Then the ring $R$ is Gorenstein (see [2, Theorem 3.1]), and hence, the projective module ${ }_{R} C$ has finite injective dimension. Also, it is clear that $C_{S}$ has finite injective dimension since $S=\mathbb{Z}[G]$ is 1 -Gorenstein. Since $\operatorname{End}_{S}(C) \varsubsetneqq R, C$ is not dualizing. However, ${ }_{R} C$ is self-small (it is countable) and $\Sigma$-self-orthogonal, and $S=\operatorname{End}_{R}(C)$ is (left and right) Noetherian and 1-perfect.

In addition, ${ }_{R} C$ is self-Hom-faithful since $\operatorname{Hom}_{R}(R(1-e), R e) \cong(1-e) R e=$ 0 and $R(1-e)$ is projective and finitely generated, so if $\operatorname{Hom}_{R}(C, X)=0$ for some $X \in \sigma[C]$, then $\operatorname{Hom}_{R}(C \oplus R(1-e), X)=0$; hence $X \cong \operatorname{Hom}_{R}(R, X)=0$ $\left(\operatorname{Hom}_{R}(R(1-e), X)=0\right.$ for all $X \in \sigma[C]$ since otherwise we would get a nonzero map $R(1-e) \rightarrow C^{(I)} / L$ and, by the projectivity of $R(1-e)$, a nonzero map $R(1-e) \rightarrow C^{(I)}$, and so a nonzero map $R(1-e) \rightarrow C$, which is impossible).

In this case $\mathcal{A}_{C}(S)=\mathbb{Z}[G]$-Mod and $\mathcal{B}_{C}(R)=\sigma[C]$.

A strong injective cogenerator of $R$-Mod is an injective left $R$-module $E$ such that any other injective $R$-module can be embedded into a direct sum of copies of $E$.

Now, we extend [5, Lemma 3.15]. It is, in some sense, a dual version of the last result.

\section{THEOREM 3.7}

Let ${ }_{R} C$ be a left $R$-module and $S=\operatorname{End}_{R}(C)$. Suppose the following.

(a) $\operatorname{inj} \cdot \operatorname{dim}_{R}(C)=m<\infty$.

(b) $C^{\vee}$ is self-Hom-cofaithful.

(c) $R$ is left Noetherian.

(d) $C_{S}$ has a degreewise finite projective resolution.

(e) proj.dim $\operatorname{dim}_{S}\left(\operatorname{Hom}_{R}(C, E)\right)=d<\infty$ for some strong injective cogenerator ${ }_{R} E$.

Then, the following conditions are equivalent.

(i) $\operatorname{Inj}(R) \subseteq \mathcal{B}_{C}(R)$.

(ii) $\mathcal{B}_{C}(R)=\{N \in R$-Mod $\mid \operatorname{Gid}(N)<\infty\}$.

(iii) (1) ${ }_{R} C$ is balanced, that is, the canonical map $R \rightarrow \operatorname{End}_{S}(C)$ is an isomorphism.

(2) $C_{S}$ is self-orthogonal, that is, $\operatorname{Ext}_{\bar{S}}^{\geq 1}(C, C)=0$.

(iv) $\mathcal{A}_{C}(S)$ consists of the modules with a finite right $C-G I(S)$ resolution. 
Proof

The proof of (ii) $\Rightarrow$ (i) is clear and (i) $\Leftrightarrow$ (iii) follows by Theorem 2.5.

(i) $\Rightarrow$ (ii) We have that $\{M \in R$-Mod $\mid \operatorname{Gid}(M)<\infty\} \subseteq \mathcal{B}_{C}(R)$ is a direct consequence of the hypothesis and Proposition 2.4(2).

For the converse let $N \in \mathcal{B}_{C}(R)$, and let

$$
0 \rightarrow N \rightarrow I_{0} \rightarrow I_{1} \rightarrow \cdots \rightarrow I_{d-1} \rightarrow I_{d} \rightarrow \cdots
$$

be an injective resolution of ${ }_{R} N$. We will see that $K_{d-1}=\operatorname{coker}\left(I_{d-2} \rightarrow I_{d-1}\right) \in$ $\mathcal{G I}(R)$.

The sequence

$$
0 \rightarrow \operatorname{Hom}_{R}(C, N) \rightarrow \operatorname{Hom}_{R}\left(C, I_{0}\right) \rightarrow \cdots
$$

is exact by $(\mathrm{B} 1)$. Let $I \in \operatorname{Inj}(R)$. Then the sequence

$$
\begin{aligned}
0 & \rightarrow \operatorname{Hom}_{S}\left(\operatorname{Hom}_{R}(C, I), \operatorname{Hom}_{R}(C, N)\right) \rightarrow \operatorname{Hom}_{S}\left(\operatorname{Hom}_{R}(C, I), \operatorname{Hom}_{R}\left(C, I_{0}\right)\right) \\
& \rightarrow \cdots
\end{aligned}
$$

is exact from the term $\operatorname{Hom}_{S}\left(\operatorname{Hom}_{R}(C, I), \operatorname{Hom}_{R}\left(C, I_{d}\right)\right)$ by (e). Also the sequence is isomorphic to

$$
0 \rightarrow \operatorname{Hom}_{R}\left(C \otimes_{S} \operatorname{Hom}_{R}(C, I), N\right) \rightarrow \operatorname{Hom}_{R}\left(C \otimes_{S} \operatorname{Hom}_{R}(C, I), I_{0}\right) \rightarrow \cdots .
$$

But since $I \in \mathcal{B}_{C}(R)$ it follows that $I \cong C \otimes_{S} \operatorname{Hom}_{R}(C, I)$, so the last sequence is isomorphic to

$$
0 \rightarrow \operatorname{Hom}_{R}(I, N) \rightarrow \operatorname{Hom}_{R}\left(I, I_{0}\right) \rightarrow \cdots,
$$

which is exact from the term $\operatorname{Hom}_{R}\left(I, I_{d}\right)$ by the above. Therefore, $\operatorname{Ext}_{R}^{i \geq d+1}(I$, $N)=0$ and so $\operatorname{Ext}_{R}^{i \geq 1}\left(I, K_{d-1}\right)=0$. We arrive at $\operatorname{Ext}_{R}^{i \geq 1}\left(V, K_{d-1}\right)=0$ for all $V$ with inj. $\operatorname{dim}_{R}(V)<\infty$.

Let $0 \rightarrow W \rightarrow E \rightarrow K_{d-1} \rightarrow 0$ be exact with $\psi: E \rightarrow K_{d-1}$ an injective cover (which is epic by the epic map $I_{d-1} \rightarrow K_{d-1}$ ). Then, by the above, $\operatorname{Ext}_{R}^{1}(V, W)=$ 0 for all $V$ with inj. $\operatorname{dim}_{R}(V)<\infty$. We consider $P \rightarrow \operatorname{Hom}_{R}(C, W) \rightarrow 0$ with $P \in$ $\operatorname{Proj}(S)$. Then $C \otimes_{S} P \rightarrow C \otimes_{S} \operatorname{Hom}_{R}(C, W) \cong W \rightarrow 0\left(\right.$ since $K_{d-1}, E \in \mathcal{B}_{C}(R)$, we have that $\left.W \in \mathcal{B}_{C}(R)\right)$ and inj. $\operatorname{dim}_{R}\left(C \otimes_{S} P\right)<\infty \operatorname{since} i n j \cdot \operatorname{dim}_{R}(C)<\infty$. If $E^{\prime} \rightarrow W$ is an injective cover, then the map $C \otimes_{S} P \rightarrow W$ factorizes through $E^{\prime} \rightarrow W$ and then it is an epimorphism. Then we can continue in the same manner with $\operatorname{ker}\left(E^{\prime} \rightarrow W\right)$ and so on. Therefore, $K_{d-1}$ has an exact left $\operatorname{Inj}(R)$-resolution and

$$
\operatorname{Ext}_{R}^{i \geq 1}\left(I, K_{d-1}\right)=0, \quad \forall I \in \operatorname{Inj}(R)
$$

so $K_{d-1} \in \mathcal{G I}(R)$.

(ii) $\Leftrightarrow$ (iv) This follows by the same ideas of the above statements.

\section{EXAMPLE 3.8}

We consider now an example of a module verifying the hypotheses of Theorem 3.7. 
Let $R$ be the triangular matrix ring

$$
R=\left(\begin{array}{cc}
\mathbb{Q}[G] & \mathbb{Q}[G] \\
0 & \mathbb{Z}[G]
\end{array}\right)
$$

where $G$ is a finite group, and let $e$ be the idempotent

$$
e=\left(\begin{array}{ll}
1 & 0 \\
0 & 0
\end{array}\right)
$$

Let

$$
C=R e=\left(\begin{array}{cc}
\mathbb{Q}[G] & 0 \\
0 & 0
\end{array}\right) .
$$

The endomorphism ring is

$$
S=e R e \cong \mathbb{Q}[G] .
$$

Then the ring $R$ is again left Noetherian Gorenstein (see [2, Theorem 3.1]), and hence, the projective module ${ }_{R} C$ has finite injective dimension. We see that $C_{S}$ is easily $\otimes_{S}$-faithful and verifies the other conditions of Theorem 3.7. However, $C$ is not balanced; hence, $\mathcal{B}_{C}(R) \neq\{N \in R$-Mod $\mid \operatorname{Gid}(N)<\infty\}$. In fact, $R$-Mod $=$ $\{N \in R$-Mod $\mid \operatorname{Gid}(N)<\infty\}$.

\section{COROLLARY 3.9}

In the conditions of Theorem 3.7, if ${ }_{R} C$ is finitely generated and self-orthogonal (i.e., $\left.\operatorname{Ext}_{R}^{i \geq 1}(C, C)=0\right)$, then the following conditions are equivalent.

(i) $\mathcal{B}_{C}(R)=\{N \in R$-Mod $\mid \operatorname{Gid}(N)<\infty\}$.

(ii) ${ }_{R} C$ is Wakamatsu tilting.

Proof

(i) $\Rightarrow$ (ii) We only have to prove the existence of an exact $\operatorname{Hom}_{R}(-, C)$-exact sequence

$$
0 \rightarrow R \rightarrow C_{0} \rightarrow C_{1} \rightarrow \cdots
$$

with $C_{i} \in \operatorname{add}_{R}(C), \forall i \geq 0$. By the hypotheses, there is an exact sequence

$$
\cdots \rightarrow Q^{1} \rightarrow Q^{0} \rightarrow C \rightarrow 0
$$

in Mod-S with $Q^{i} \in \operatorname{Proj}(S)$ and finitely generated $\forall i \geq 0$. We will check that

$$
0 \rightarrow \operatorname{Hom}_{S}(C, C) \cong R \rightarrow \operatorname{Hom}_{S}\left(Q^{0}, C\right) \rightarrow \operatorname{Hom}_{S}\left(Q^{1}, C\right) \rightarrow \cdots
$$

is our desired sequence. It is easy to see that the sequence is exact by the condition $\operatorname{Ext}_{S}^{i \geq 1}(C, C)=0$ (see Theorem 3.7). Now, from the commutative diagram

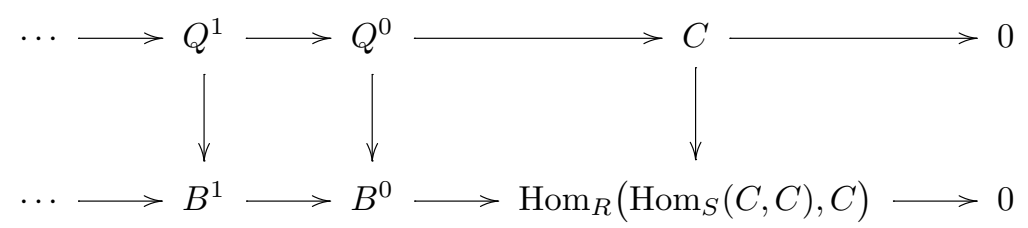


where $B^{i}=\operatorname{Hom}_{R}\left(\operatorname{Hom}_{S}\left(Q^{i}, C\right), C\right)$, we deduce that the sequence is $\operatorname{Hom}_{R}(-$, $C)$-exact since the vertical maps are isomorphisms.

(ii) $\Rightarrow$ (i) By [14, Corollary 3.2] $C_{S}$ is Wakamatsu tilting with $R \cong \operatorname{End}_{S}(C)$. Then by (iii) $\Rightarrow$ (ii) in Theorem 3.7 we get the result.

Also, we can extend [10, Remark 3.13] to our generality without too much effort. We leave the proof to the reader.

\section{COROLLARY 3.10 (FOXBY EQUIVALENCES)}

In the conditions of Theorems 3.4 and 3.7, we have the following equivalences of categories:

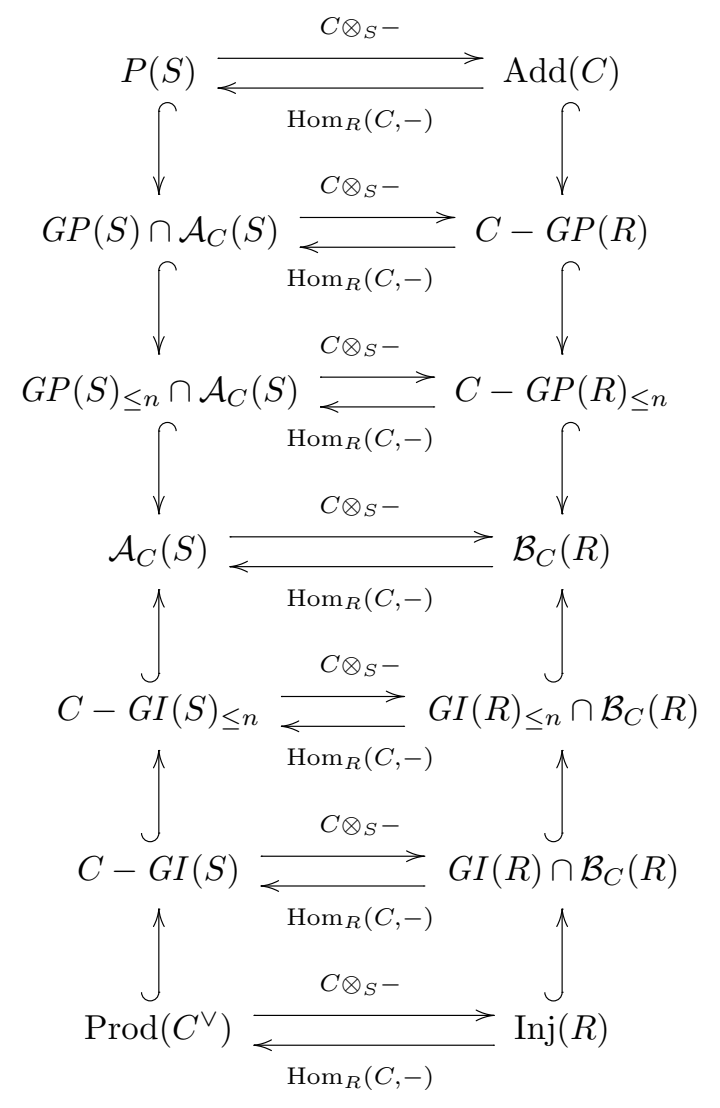

where $G P(S)_{\leq n}$ denotes the class of modules with $G P(S)$-dimension less than or equal to $n$ and the other classes are given analogously.

\section{When is $\mathcal{A}_{C}(S)\left(\mathcal{B}_{C}(R)\right)$ covering (enveloping)?}

The following results follow easily from [9, Theorems 2.6 and 2.9]. Let

$$
\begin{aligned}
\operatorname{Inj}_{C}(S) & =\left\{\operatorname{Hom}_{R}(C, E) \mid E \in \operatorname{Inj}(R)\right\} \subseteq S \text {-Mod, } \\
\operatorname{Proj}_{C}(R) & =\left\{C \otimes_{S} P \mid P \in \operatorname{Proj}(S)\right\} \subseteq R \text {-Mod, }
\end{aligned}
$$




$$
\text { Flat }_{C}(R)=\left\{C \otimes_{S} F \mid F \in \operatorname{Flat}(S)\right\} \subseteq R \text {-Mod. }
$$

Note that $\operatorname{Add}_{R}(C)=\operatorname{Proj}_{C}(R)$.

\section{THEOREM 4.1}

Let $C$ be a left $R$-module, and let $S=\operatorname{End}_{R}(C)$. Suppose $\operatorname{Inj}(R) \subseteq \mathcal{B}_{C}(R)$.

The following assertions are equivalent for $M \in S$-Mod.

(i) $\quad M \in \mathcal{A}_{C}(S)$.

(ii) There is an exact sequence

$$
\cdots F^{1} \rightarrow F^{0} \rightarrow U_{0} \rightarrow U_{1} \rightarrow \cdots
$$

in $S$-Mod, where every $F_{i}$ is flat and every $U_{i} \in \operatorname{Inj}_{C}(S)$, such that $M=\operatorname{ker}\left(U_{0} \rightarrow\right.$ $\left.U_{1}\right)$ and which remains exact when $C \otimes_{S}-$ is applied.

(iii) There is an exact sequence

$$
0 \rightarrow M \rightarrow U_{0} \rightarrow U_{1} \rightarrow \cdots
$$

in $S$-Mod, where every $U_{i} \in \operatorname{Inj}_{C}(S)$, such that it remains exact when $\operatorname{Hom}_{S}(-, U)$ is applied for every $U \in \operatorname{Inj}_{C}(S)$ and $C \otimes_{S}-$ leaves exact every flat resolution of $M$.

\section{THEOREM 4.2}

Let $C$ be a left $R$-module, and let $S=\operatorname{End}_{R}(C)$. Suppose $\operatorname{Proj}(S) \subseteq \mathcal{A}_{C}(S)$.

The following assertions are equivalent for $N \in R$-Mod.

(i) $\quad N \in \mathcal{B}_{C}(R)$.

(ii) There is an exact sequence

$$
\rightarrow \cdots W^{1} \rightarrow W^{0} \rightarrow E_{0} \rightarrow E_{1} \rightarrow \cdots
$$

in $R$-Mod, where every $E_{i}$ is injective and $W^{i} \in \operatorname{Flat}_{C}(R)$, such that $\operatorname{Hom}_{R}(C,-)$ leaves it exact and $N=\operatorname{ker}\left(E_{0} \rightarrow E_{1}\right)$.

(iii) There is an exact Flat $_{C}(R)$-resolution

$$
\cdots \rightarrow W^{1} \rightarrow W^{0} \rightarrow N \rightarrow 0
$$

such that it remains exact when $\operatorname{Hom}_{R}(W,-)$ is applied for every $W \in \operatorname{Flat}_{C}(R)$ and $\operatorname{Hom}_{R}(C,-)$ leaves exact every injective resolution of $N$.

\section{PROPOSITION 4.3}

(a) Suppose $C^{\vee}$ is self-Hom-cofaithful and $\operatorname{Inj}(R) \subseteq \mathcal{B}_{C}(R)$. Then we have the following.

(i) $\operatorname{Inj}_{C}(R)$ is closed under pure submodules and pure quotients.

(ii) $\mathcal{B}_{C}(R)$ is a Kaplansky class.

(b) Suppose ${ }_{R} C$ is self-Hom-faithful and finitely presented and $\operatorname{Flat}(S) \subseteq$ $\mathcal{A}_{C}(S)$. Then we have the following.

(iii) Flat $_{C}(R)$ is closed under pure submodules and pure quotients.

(iv) $\mathcal{A}_{C}(S)$ is a Kaplansky class. 
Proof

Parts (i) and (iii) follow by the arguments in the proof of [3, Proposition 3.9]. Parts (ii) and (iv) can be proved using the same proof as that of [3, Proposition 3.10].

As a direct consequence of the above, we can show the existence of precovers and preenvelopes of the Auslander and Bass classes in this general setting.

\section{THEOREM 4.4}

(a) Suppose ${ }_{R} C$ has a degreewise projective resolution, it is self-Hom-faithful, and $\operatorname{Flat}(S) \subseteq \mathcal{A}_{C}(S)$. Then, $\left(\mathcal{A}_{C}(S), \mathcal{A}_{C}(S)^{\perp}\right)$ is a perfect cotorsion pair; in particular, $\mathcal{A}_{C}(S)$ is covering. If, in addition, $C_{S}$ has a degreewise projective resolution, then $\mathcal{A}_{C}(S)$ is preenveloping.

(b) Suppose $C^{\vee}$ is self-Hom-cofaithful, $\operatorname{Inj}(R) \subseteq \mathcal{B}_{C}(R),{ }_{R} C$ has a degreewise projective resolution, and $C_{S}$ has a degreewise projective resolution. Then $\mathcal{B}_{C}(R)$ is preenveloping.

Proof

See [3, Theorems 3.11 and 3.12].

We end this article by answering the question of when the classes of modules with finite Gorenstein projective dimension (resp., Gorenstein injective dimension) are precovering (resp., preenveloping). We extend [3, Corollary 3.13] to the noncommutative setting.

\section{THEOREM 4.5}

Let $R$ be a ring such that there is a left $R$-module ${ }_{R} C$ with $S=\operatorname{End}_{R}(C)$ verifying:

(a) inj. $\operatorname{dim}_{R}(C)<\infty$ and inj. $\operatorname{dim}_{S}(C)<\infty$,

(b) ${ }_{R} C$ is self-Hom-faithful,

(c) $S$ is right Noetherian and left n-perfect,

(d) ${ }_{R} C$ is self-orthogonal,

(e) ${ }_{R} C$ has a degreewise projective resolution.

Then $\{M \in S-\operatorname{Mod} \mid \operatorname{Gpd}(M)<\infty\}$ is covering. If, in addition, $C_{S}$ is finitely generated, then $\{M \in S$-Mod $\mid \operatorname{Gpd}(M)<\infty\}$ is preenveloping.

Proof

It is a direct consequence of Theorems 3.4 and 4.4 .

THEOREM 4.6

Let $R$ be a left Noetherian ring such that there is a finitely generated left $R$-module ${ }_{R} C$ with $S=\operatorname{End}_{R}(C)$ and $\operatorname{End}_{S}(C) \cong R$ verifying: 
(a) inj. $\operatorname{dim}_{R}(C)=m<\infty$,

(b) $C^{\vee}$ is self-Hom-cofaithful,

(c) $C_{S}$ has a degreewise finite projective resolution,

(d) proj.dim $\operatorname{dim}_{S}\left(\operatorname{Hom}_{R}(C, E)\right)<\infty$ for some strong injective cogenerator ${ }_{R} E$,

(e) $C_{S}$ is self-orthogonal, that is, $\operatorname{Ext}_{\bar{S}}^{\geq 1}(C, C)=0$.

Then $\{M \in R$-Mod $\mid \operatorname{Gid}(M)<\infty\}$ is preenveloping.

Proof

This follows from Theorems 3.7 and 4.4.

Acknowledgment. This work started when the first author was visiting the University of Almería.

\section{References}

[1] D. Bennis, J. R. García Rozas, and L. Oyonarte, Relative Gorenstein dimensions, Mediterr. J. Math. 13 (2016), 65-91. MR 3456908. DOI 10.1007/s00009-014-0489-8.

[2] E. E. Enochs, M. Cortés-Izurdiaga, and B. Torrecillas, Gorenstein conditions over triangular matrix rings, J. Pure Appl. Algebra 218 (2014), 1544-1554. MR 3175039. DOI 10.1016/j.jpaa.2013.12.006.

[3] E. E. Enochs and H. Holm, Cotorsion pairs associated with Auslander categories, Israel J. Math. 174 (2009), 253-268. MR 2581218. DOI 10.1007/s11856-009-0113-y.

[4] E. E. Enochs and O. M. G. Jenda, Relative Homological Algebra, De Gruyter Exp. Math. 30, de Gruyter, Berlin, 2000. MR 1753146. DOI $10.1515 / 9783110803662$.

[5] E. E. Enochs, O. M. G. Jenda, and J. A. López-Ramos, Dualizing modules and n-perfect rings, Proc. Edinb. Math. Soc. (2) 48 (2005), 75-90. MR 2117713. DOI 10.1017/S0013091503001056.

[6] E. E. Enochs, O. M. G. Jenda, and J. Z. Xu, Foxby duality and Gorenstein injective and projective modules, Trans. Amer. Math. Soc. 348, no. 8 (1996), 3223-3234. MR 1355071. DOI 10.1090/S0002-9947-96-01624-8.

[7] E. E. Enochs and S. Yassemi, Foxby equivalence and cotorsion theories relative to semi-dualizing modules, Math. Scand. 95 (2004), 33-43. MR 2091480.

[8] H.-B. Foxby, Quasi-perfect modules over Cohen-Macaulay rings, Math. Nachr. 66 (1975), 103-110. MR 0376663.

[9] J. R. García Rozas, J. A. López-Ramos, and B. Torrecillas, Semidualizing and tilting adjoint pairs, applications to comodules, Bull. Malays. Math. Sci. Soc. 38 (2015), 197-218. MR 3394048. DOI 10.1007/s40840-014-0013-0.

[10] Y. Geng and N. Ding, W-Gorenstein modules, J. Algebra 325 (2011), 132-146. MR 2745532. DOI 10.1016/j.jalgebra.2010.09.040. 
[11] H. Holm and D. White, Foxby equivalence over associative rings, Kyoto J. Math. 47 (2007), 781-808. MR 2413065.

[12] T. Ishikawa, On injective modules and flat modules, J. Math. Soc. Japan 17 (1965), 291-296. MR 0188272.

[13] J. Rada and M. Saorin, Rings characterized by (pre)envelopes and (pre)covers of their modules, Comm. Algebra 26 (1998), 899-912. MR 1606190. DOI 10.1080/00927879808826172.

[14] T. Wakamatsu, Tilting modules and Auslander's Gorenstein property, J. Algebra 275 (2004), 3-39. MR 2047438. DOI 10.1016/j.jalgebra.2003.12.008.

[15] R. Wisbauer, "Cotilting objects and dualities" in Representations of Algebras (São Paulo, 1999), Lecture Notes in Pure and Appl. Math. 224, Dekker, New York, 2002, 215-233. MR 1884820.

Bennis: Department of Mathematics, Faculty of Sciences, Mohammed V. University, Rabat, Morocco; d.bennis@fsr.ac.ma; driss_bennis@hotmail.com

García Rozas: Departamento de Matemáticas, Universidad de Almería, Almería, Spain; jrgrozas@ual.es

Oyonarte: Departamento de Matemáticas, Universidad de Almería, Almería, Spain; oyonarte@ual.es 